\title{
Correction to: The Ryan White HIV-AIDS Program: Supplementary Service Provision Post-Affordable Care Act, by Kay ES, Batey DS, and Mugavero MJ. AIDS Patient Care STDs 2018;32:265-271. DOI: 10.1089/apc.2018.0032
}

T the Article, "The Ryan White HIV-AIDS Program: Supplementary Service Provision Post-Affordable Care Act," published in AIDS Patient Care STDs 2018;32:265-271, there were two errors on page 267.

The first error was in Table 1, where it showed that 525 patients have RWHAP supplementary services and 643 do not. This is correct, but the percentages related to these values need to be switched-54.7\% should be with 643 patients, and $45.3 \%$ should be with 525 patients. Table 1 is reproduced here with the correct data, and it has been corrected in the online article.

The second error was also on p. 267, in the right column, under Results, in the following sentence: "Half (52.4\%) of patients reported a high school degree or its equivalent as the highest education level attained, and the mean income was $\$ 963.77$ per month (ranging from $\$ 0$ to $\$ 8000$ ) (Table 2)." This sentence should refer to Table 1 instead of Table 2; also, the monthly income range should be $\$ 0-\$ 5572$, instead of $\$ 0-\$ 8000$, as is correctly displayed in Table 1 . The correct sentence is as follows: "Half (52.4\%) of patients reported a high school degree or its equivalent as the highest education level attained, and the mean income was $\$ 963.77$ per month (ranging from $\$ 0$ to $\$ 5572$ ) (Table 1)." This sentence has been corrected in the online article.

The author regrets these errors.

Table 1. Characteristics for Patients Receiving HiV Primary Care Services in 2016

\begin{tabular}{lcc}
\hline Characteristic & $\mathrm{n}(\%)$ & Mean (SD); min.- max. \\
\hline RWHAP Supplementary & \\
Yes & $525(45.3)$ \\
No & $643(54.7)$ \\
Primary payer type & $659(56.9)$ \\
Private Insurance & $379(32.7)$ \\
Public Insurance & $44(3.8)$ \\
RWHAP & $77(6.6)$ \\
Uninsured (no RWHAP) & \\
Combination payer type & $157(13.5)$ \\
Public insurance + RWHAP & $266(23.0)$ \\
Private insurance + RWHAP & $222(19.2)$ \\
Public insurance only & $393(33.9)$ \\
Private insurance only & $102(8.8)$ \\
RWHAP only & $19(1.6)$ \\
Uninsured (no RWHAP) & \\
Gender & $835(72.0)$ \\
Male & $324(28.0)$ \\
Female & \\
Race/ethnicity & $897(77.4)$ \\
Black & $221(19.1)$ \\
White & $41(3.5)$ \\
Other &
\end{tabular}


TABle 1. (CONTINUED)

\begin{tabular}{lcc}
\hline Characteristic & $\mathrm{n}(\%)$ & Mean (SD); min.- max. \\
\hline Monthly income & 1159 & $\$ 963.77(\$ 804.86) \$ 0-\$ 5572$ \\
Age (in years) & 1159 & $44.4(12.5) 20-83$ \\
Highest ed. level & $264(22.8)$ & $607(52.4)$ \\
$\quad$ Less than HS & $190(16.4)$ \\
High school or GED & $98(8.5)$ \\
Some college & $1059(91.4)$ \\
College or higher & $100(8.6)$ \\
Viral suppression & $1022(87.4)$ \\
Yes & $147(12.6)$ \\
No & \\
Retention in care: IOM kept-visits & $698(60.0)$ \\
Yes & $461(40.0)$ \\
No &
\end{tabular}

Percentages may not sum to 100 due to rounding. 